\title{
Transport in a Luttinger liquid with dissipation: two impurities
}

\author{
Leiming Chen, Zoran Ristivojevic, and Thomas Nattermann \\ Institut für Theoretische Physik, Universität zu Köln, Zülpicher Str. 77, 50937 Köln, Germany
}

(Dated: 02-11-2018 03:34)

\begin{abstract}
We consider theoretically the transport in a one-channel spinless Luttinger liquid with two strong impurities in the presence of dissipation. As a difference with respect to the dissipation free case, where the two impurities fully transmit electrons at resonance points, the dissipation prevents complete transmission in the present situation. A rich crossover diagram for the conductance as a function of applied voltage, temperature, dissipation strength, Luttinger liquid parameter $K$ and the deviation from the resonance condition is obtained. For weak dissipation and $1 / 2<K<1$, the conduction shows a non-monotonic increase as a function of temperature or voltage. For strong dissipation the conduction increases monotonically but is exponentially small.
\end{abstract}

PACS numbers: 71.10.Pm,72.10.Bg,72.80.Ng,73.21.Hb

\section{INTRODUCTION}

Once a quantum mechanical particle traveling in onedimensional space hits a potential barrier formed by an impurity, it is reflected with a finite probability. This is not longer true in the case of two consecutive identical barriers forming a quantum dot: the waves reflected at the first and the second barrier may interfere destructively such that there is perfect transmission. The condition for the latter reads $\tan (k a)=-\hbar^{2} k /\left(m U_{0}\right)$. Here $m$ and $k$ denotes the mass and the wavevector of the incoming wave, and $a$ is the spacing between the two barriers which are here assumed to be delta functions of strength $U_{0}$. For small and large $U_{0}$, respectively, this resonance corresponds to the conditions $k a=(n+1 / 2) \pi$ and $k a=(n+1) \pi$, respectively, where $n \geq 0$ is integer. Thus, for large $U_{0}$ the resonance happens if the incident particle has the same energy as one of the bound states formed between the barriers, provided they are impenetrable.

So far we considered free electrons. In this paper we will examine the case of interacting electrons in one dimension where the interaction can be parametrized by a single parameter $K$ with $K>1(K<1)$ for repulsive (attractive) interaction 1.2 . Then, in the case of scattering at an isolated impurity, attractive (repulsive) interaction leads to perfect transmission (reflection) ${ }^{3}$ at low energies.

If two consecutive strong impurities are present, the physics is influenced by the Coulomb blockade in the quantum dot. As was shown by Kane and Fisher 4,5 and by Furusaki and Nagaosa ${ }^{6}$, resonant transmission of (spinless) electrons is then possible provided $k_{F} a=$ $\left(n+\frac{1}{2}\right) \pi$, where $k_{F}$ is the Fermi momentum and $n \geq 0$ integer. With $k_{F} a / \pi \equiv q_{0}$ the background charge between the two impurities, at resonance the ground state of the dot is doubly degenerate with $n_{ \pm}=q_{0} \pm 1 / 2$ particles inside the dot. Note that this resonance condition is independent of the impurity strength $U_{0}$. Tunneling at resonance is sequential. If one starts with $n_{-}$state of the dot, adding a particle to the dot does not change the Coulomb energy. In a second tunneling step the particle number in the dot goes back to $n_{-}$. In a similar way we may start with the $n_{+}$state and then first decrease $n_{+}$ by one, which is followed by a second electron tunneling into the dot to get back to the original state $n_{+}$.

Thus under resonance conditions adding (or removing) and electron does not change the Coulomb energy inside the dot. Renormalization group analysis of the impurity strength then shows that perfect transmission is still present for weak impurities as long as $K>1 / 4^{4,5,6}$. For strong impurities perfect transmission survives for $K>1 / 2$. The conductance in both cases is given by $e^{2} / h$ for spinless electrons. Therefore, similar to the noninteracting case, the existence of a second impurities increases the tendency to perfect transmission for not too strong repulsive interaction.

Adding an electron off resonance is accompanied by an energy increase which has to be provided either by a thermal bath or by a finite external voltage. A thermal bath as well as a finite voltage drop across the dot also allows sequential tunneling off-resonance, which leads to power laws of the conductance as a function of temperature or voltage. If both are small enough, sequential tunneling is suppressed and tunneling occurs in one step via the formation of a virtual state in the dot (co-tunneling).

In the present paper we want to study the influence of ohmic dissipation on the scenario presented so far. As discussed recently in $\operatorname{Ref} \stackrel{7}{\underline{7}}$, ohmic dissipation may result from the coupling of electrons in the Luttinger liquid to normal Fermi liquid like electrons in nearby gates. Under the conditions considered in $\underline{7}^{\underline{7}}$ coupling to the gate is relevant only for $K<K_{\eta}=1 / 2$. However, other scenarios are conceivable, and in the following we will assume that $K_{\eta}$ may take also larger values. We will therefore assume that dissipation is present. Clearly, for $K>K_{\eta}$ our results have to be replaced by those of the dissipation free case. of the wire obeys Dissipation introduces a new length scale $L_{\eta} \approx 1 /(K \eta)$ where $\eta$ denotes the dissipation strength. On scales larger than $L_{\eta}$ the plasmon excitations of the electrons become diffusive and displacement fluctuations are strongly suppressed, restoring translational long range order (Wigner crystal). If $K v \eta \gg \omega$, the conductivity $\sigma_{\eta}=2 K L_{\eta} e^{2} / h$ is finite which is paralleled by diverging superfluid fluctuations. 
We have recently shown ${ }^{8}$ that dissipation has a dramatic influence on the tunneling of electrons through a single impurity, which is strongly suppressed. The voltage and temperature dependence of the conductance is reduced from power laws in the dissipation free case to an exponential dependence for all $K<K_{\eta}$. Thus there is no region of perfect transmission anymore with a single impurity. In the present paper we want to extend these considerations to the case of two impurities.

The paper is organized as follows. In Sec. II we introduce a model for spinless electrons in the presence of dissipation and with two impurities. Using the instanton method, we study the electron tunneling through impurities driven by external voltage or temperature. The conductance of the system is calculated in Sec. III for the co-tunneling case, and in Sec. IV for the sequential tunneling. Conclusions are drawn in Sec. V. Some technical details are presented in the appendices.

\section{THE TUNNELING PROBABILITY}

\section{A. The model}

We consider a one-dimensional interacting system of spinless electron with two impurities coupled to a dissipative bath. The impurities are at positions $x= \pm a / 2$, respectively. We will refer to the spacing between the impurities as the quantum dot. Using the standard bosonization methods 2.9 , the Euclidean action for this system is

$$
\begin{aligned}
\frac{S}{\hbar}= & \int_{-\frac{L}{2}}^{\frac{L}{2}} d x \int_{-\frac{\hbar \beta}{2}}^{\frac{\hbar \beta}{2}} d \tau\left\{\frac{1}{2 \pi K}\left[\frac{1}{v}\left(\partial_{\tau} \phi\right)^{2}+v\left(\partial_{x} \phi\right)^{2}\right]\right. \\
& \left.+\rho \frac{U_{0}}{\hbar}[\delta(x-a / 2)+\delta(x+a / 2)]\right\} \\
& +\frac{\eta}{4} \int_{-\frac{L}{2}}^{\frac{L}{2}} d x \int_{-\frac{\hbar \beta}{2}}^{\frac{\hbar \beta}{2}} d \tau \int_{-\frac{\hbar \beta}{2}}^{\frac{\hbar \beta}{2}} d \tau^{\prime} \frac{\left[\phi(x, \tau)-\phi\left(x, \tau^{\prime}\right)\right]^{2}}{\left[\hbar \beta \sin \frac{\pi\left(\tau-\tau^{\prime}\right)}{\hbar \beta}\right]^{2}}
\end{aligned}
$$

Here the displacement field $\phi(x, \tau)$ is related to the electron density $\rho$ by

$$
\rho=\frac{k_{F}}{\pi}-\frac{1}{\pi} \partial_{x} \phi+\frac{k_{F}}{\pi} \cos \left(2 \phi-2 k_{F} x\right)+\ldots
$$

The first term of action (11) is the well-known TomonagaLuttinger model. The parameter $K$ measures the interactions between electrons, where $K<1$ for attractive interactions and $K>1$ for repulsive interactions, respectively. $v$ is the velocity of the plasmon excitations. The second part in action (11) is the contributions from the two impurities, where we have assumed that the two impurities have the same strength $U_{0}$.

The third piece describes Ohmic dissipation $\underline{10}$. It was shown in Ref $\mathbb{7}^{\underline{7}}$ that a dissipation term of the form

$$
-\frac{\eta}{2} \int_{-L / 2}^{L / 2} d x \int_{-\frac{\hbar \beta}{2}}^{\frac{\hbar \beta}{2}} d \tau \int_{-\frac{\hbar \beta}{2}}^{\frac{\hbar \beta}{2}} d \tau^{\prime} \frac{\cos \left[\phi(x, \tau)-\phi\left(x, \tau^{\prime}\right)\right]}{\left[\hbar \beta \sin \frac{\pi\left(\tau-\tau^{\prime}\right)}{\hbar \beta}\right]^{2}}
$$

results from the coupling of the electrons in the wire to Fermi fluid electrons in a nearby gate, where $\eta$ is a coupling constant. When the coupling is relevant, Eq. (3) can be expanded up to quadratic terms, and it has been done in Eq. (1).

Integrating out the bulk phase field $\phi(x, \tau)$ except $\phi(x=-a / 2, \tau)$ and $\phi(x=a / 2, \tau)$, we obtain the effective action as

$$
\begin{aligned}
\frac{S}{\hbar}= & \frac{1}{2 K \hbar \beta} \sum_{\omega_{n}}\left[\frac{1}{I_{+}\left(\omega_{n}\right)}\left|\phi_{+}\left(\omega_{n}\right)\right|^{2}+\frac{1}{I_{-}\left(\omega_{n}\right)}\left|\phi_{-}\left(\omega_{n}\right)\right|^{2}\right] \\
& +U \int d \tau \cos \left[\phi_{+}(\tau)\right] \cos \left[\phi_{-}(\tau)-k_{F} a\right]
\end{aligned}
$$

where

$$
\begin{aligned}
& \phi_{ \pm}(\tau)=\phi(a / 2, \tau) \pm \phi(-a / 2, \tau), \\
& \phi_{ \pm}\left(\omega_{n}\right)=\int d \tau e^{i \omega_{n} \tau} \phi_{ \pm}(\tau), \quad \omega_{n}=\frac{2 \pi n}{\hbar \beta}, \\
& I_{ \pm}\left(\omega_{n}\right)=\frac{\pi\left(1 \pm e^{-\frac{a}{v} \sqrt{\omega_{n}^{2}+\left|\omega_{n}\right| v \eta K}}\right)}{\sqrt{\omega_{n}^{2}+\left|\omega_{n}\right| v \eta K}} .
\end{aligned}
$$

$U=2 k_{F} U_{0} /(\pi \hbar)$ denotes the dimensionless pinning strength.

\section{B. Classical Ground State and Excitations}

We will first look at the classical ground state, where $\phi(x, \tau) \equiv \phi(x)$ corresponding to weak quantum fluctuation limit $K \ll 1$. In fact our further calculation is strictly justified only in this case although we will also apply our results for $K=\mathcal{O}(1)$. The field $\phi_{-}$is related to the charge $Q$ (in units of the elementary charge) accumulated between the two impurities by

$$
Q=\int_{-a / 2}^{a / 2} d x \rho(x)=q_{0}-\frac{\phi_{-}}{\pi},
$$

where $q_{0}=k_{F} a / \pi$ denotes the background charge between the impurities. From (1), we now obtain

$$
\frac{S_{\text {class. }}}{\hbar}=\frac{E_{c}}{2 T}\left(Q-q_{0}\right)^{2}+\frac{2 k_{F} U_{0}}{\pi T} \cos \phi_{+} \cos (\pi Q),
$$

where the Coulomb energy of the quantum dot is

$$
E_{c}=\frac{1}{\kappa a}, \quad \kappa=\frac{K}{\pi v \hbar}
$$


where $\kappa$ denotes the compressibility. In the ground state, the action (9) has to be minimal. For the rest of the paper we will assume strong impurities $U \gg v k_{F}$, i.e. $U_{0} / \hbar v \gg 1$, in agreement with the strong impurity condition from Eq. (1). As a result $Q$ has to be an integer. Then, minimizing the first term in (9), one obtains $Q=Q_{0}=\left[q_{0}\right]_{G}$, where $[x]_{G}$ denotes the closest integer to $x$. Minimizing the second term gives $\phi_{+}=(Q+2 m+1) \pi$, where $m$ is an integer. The corresponding ground state values for $\phi( \pm a / 2)$ are

$$
\begin{aligned}
& \phi(-a / 2)=\frac{1}{2}\left[(2 m+1) \pi-k_{F} a\right], \\
& \phi(a / 2)=\frac{1}{2}\left[(2 m+1) \pi+k_{F} a\right]+\pi Q .
\end{aligned}
$$

In the following it is convenient to express the background charge in the dot as

$$
q_{0}=n+\frac{1}{2}-\Delta,
$$

where $n$ is an integer and $-1 / 2<\Delta<1 / 2$. This gives $Q_{0}=n$ if $\Delta>0$ and $Q_{0}=n+1$ if $\Delta<0$. For $\Delta=0$ the ground state is twofold degenerate, allowing $Q_{0}=n$ and $Q_{0}=n+1$, respectively.

As already observed by Kane and Fisher ${ }^{4}$ the action (9) remains invariant under the transformation $\phi_{+} \rightarrow$ $\phi_{+}+2 \pi$. This transformation corresponds to the transfer of one electron from the left to the right LL lead. If $q_{0}$ is exactly half-integer, i.e. $\Delta=0$, there is an additional invariance under the transformation $\phi_{+} \rightarrow \phi_{+}+\pi$ and $Q \rightarrow 2 q_{0}-Q$. This transformation corresponds to the transfer of one electron half-way across the double barrier structure. This allows the sequential tunneling through the quantum dot.

The energy cost for adding (removing) an electron to (from) the ground state is given by

$$
E_{ \pm}=\frac{E_{c}}{2}\left[1 \pm 2\left(Q_{0}-q_{0}\right)\right]=E_{c}\left[ \pm \Delta+\Theta_{H}(\mp \Delta)\right],
$$

where $\Theta_{H}(x)$ denotes the Heaviside step function.

Let us now consider a sequential tunneling process in the case $1 \gg|\Delta|>0$. For $\Delta>0$ the ground state is given by $Q_{0}=n$. To transfer an electron through the quantum dot it first goes from the left lead to the dot, which requires an energy $E_{c} \Delta$. In a second process it tunnels to the right lead which sets this energy again free. For $\Delta<0$ the ground state of the dot is $Q_{0}=n+1$ and the particle transfer begins with the tunneling of an electron from the dot to the right lead which costs $-\Delta E_{c}>0$ followed by the tunneling of an electron from the left lead to the dot. Thus, for sequential tunneling with $\Delta \neq 0$ there is always one hard tunneling step which requires an energy $|\Delta| E_{c}$. This Coulomb blockade plays a role at non-zero temperatures $T$ or voltages $V_{0}$ as long as $T, e V_{0}<E_{C}|\Delta|$. Because of the symmetry between the cases $\Delta>0$ and $\Delta<0$, we can restrict ourselves in the following to the case $\Delta>0$.

In addition to the charged excitations there are also neutral excitations in the quantum dot of spacing $\pi \hbar v / a=K /(\kappa a)=K E_{c}$. Their maximum energy is $\hbar \omega_{c} \approx \hbar v \Lambda$ where $\Lambda$ is of the order of the Fermimomentum $k_{F}$. If the temperature $T$ is larger than $K E_{c}$ the quantization of the neutral excitations in the quantum dot becomes irrelevant and the tunneling through the two impurities will become independent, i.e. the tunneling is incoherent sequential. Alternatively, one can say that the coherence length $L_{T}=\hbar v / T$ of the displacements of the electrons in the quantum dot is smaller than $a$. In the opposite limit the tunneling is coherent. In the following we will concentrate on this case.

In our model the neutral excitations are damped. Plasmons become diffusive on scales larger than $L_{\eta}=$ $1 /(K \eta)$, having a characteristic life time $L_{\eta} / v$. Phase coherence in the quantum dot is lost if $L_{\eta}<a$, i.e. if $K E_{c}<\Gamma=\hbar v K \eta$ where $\Gamma$ denotes the imaginary part of the plasmon energy.

\section{Instanton action}

The tunneling rate $\mathcal{R}$ through the impurity and hence the current $I=e \mathcal{R}$ can be calculated from the imaginary part of the free energy

$$
\mathcal{R}=\frac{2}{\hbar \beta} \operatorname{Im} \ln Z,
$$

where the partition function is

$$
Z=Z_{0}+i Z_{1}=\int \mathcal{D} \phi_{+}(\tau) \mathcal{D} \phi_{-}(\tau) e^{-S\left[\phi_{+}, \phi_{-}\right] / \hbar} .
$$

Real part of $Z$ includes the (stable) fluctuations around the classical ground state. Since the imaginary part $Z_{1}$ of the partition function is small compared with the real part $Z_{0}$, the tunneling rate can be written as

$$
\mathcal{R} \approx \frac{2}{\hbar \beta Z_{0}} \operatorname{Im} \int \mathcal{D} \phi_{+}(\tau) \mathcal{D} \phi_{-}(\tau) e^{-S\left[\phi_{+}, \phi_{-}\right] / \hbar},
$$

where the functional integral is with respect to the functions $\phi_{ \pm}(\tau)$ defined on the interval $[-\hbar \beta / 2, \hbar \beta / 2]$ and satisfying $\phi_{ \pm}(-\hbar \beta / 2)=\phi_{ \pm}(\hbar \beta / 2)$. To calculate $Z_{1}$ we follow a method developed by Callan and Coleman 11 . There exist saddle point functions $\tilde{\phi}_{ \pm}(\tau)$ which obey the equations

$$
\left.\frac{\delta S\left[\phi_{+}, \phi_{-}\right]}{\delta \phi_{ \pm}}\right|_{\phi=\tilde{\phi}}=0
$$

With $\phi_{ \pm}(\tau)=\tilde{\phi}_{ \pm}(\tau)+\delta \phi_{ \pm}(\tau)$ the action close to the saddle point trajectory can be written in the form 


$$
S\left[\phi_{+}, \phi_{-}\right]-\left.S\left[\tilde{\phi}_{+}, \tilde{\phi}_{-}\right] \approx \sum_{i, j= \pm} \frac{1}{2} \int d \tau d \tau^{\prime} \frac{\delta^{2} S}{\delta \phi_{i}(\tau) \delta \phi_{j}\left(\tau^{\prime}\right)}\right|_{\phi=\tilde{\phi}} \delta \phi_{i}(\tau) \delta \phi_{j}\left(\tau^{\prime}\right)=\sum_{\substack{i, j= \pm n, m}} V_{i, n ; j, m} a_{n, i} a_{m, j}=\sum_{n} \lambda_{n} c_{n}^{2}
$$

where in the last step we have expanded $\delta \phi_{ \pm}(\tau)$ into a complete set of orthogonal functions $\psi_{n}(\tau)$, $\delta \phi_{ \pm}(\tau)=\sum_{n} a_{n, \pm} \psi_{n}(\tau)$ and then diagonalized the resulting quadratic form in the $a_{n, \pm}$. One of the eigenvalues, $\lambda_{n=0}$, has to be negative to give rise to the imaginary part. Thus

$$
i Z_{1}=e^{-S\left[\tilde{\phi}_{+}, \tilde{\phi}_{-}\right] / \hbar} \mathcal{N} \int\left(\prod_{n} d c_{n}\right) e^{-\sum_{n} \lambda_{n} c_{n}^{2} / \hbar}
$$

One of the eigenvalue has to be zero corresponding to a shift of the instanton in the $\tau$ direction ${ }^{12}$ delivering a factor $\hbar \beta . \quad Z_{0}$ can be calculated in the same way with only positive eigenvalues at the stable saddle point.

Performing this program is very difficult in the present case. Instead, we will look for an approximate solution. In this case the saddle point function $\tilde{\phi}( \pm a / 2, \tau)$ will assume their groundstate values (11) everywhere apart from the regions where $\tilde{\phi}( \pm a / 2, \tau)$ is increases by $\pi$. This advancement of $\pi$ is triggered by the applied external voltage. The connection between these pieces are narrow kinks and anti-kinks of width $\delta \sim 1 / U \ll\left(v k_{F}\right)^{-1}$. Thus the saddle point configuration $\tilde{\phi}_{ \pm}$is determined in our approximate scheme by the positions of the kinks and anti-kinks. A kink-anti-kink pair will be called an instanton in the following.

It is sufficient to consider the case when there is only one instanton at each impurity with kink-anti-kink spacing equal to $\tau_{1}$ and $\tau_{2}$ on the left and the right impurity, respectively. To minimize the action we will assume that the centers of the instantons have the same value of $\tau$. With our parametrization the saddle point $\tilde{\phi}_{ \pm}$is now found from the condition for the instanton action $\partial S_{\text {inst }}\left(\tau_{1}, \tau_{2}\right) /\left.\partial \tau_{1,2}\right|_{\tau_{i}=\tau_{i, s}}=0$. This gives

$$
\begin{aligned}
I= & e \mathcal{R} \approx 2 e^{-S_{\mathrm{inst}}\left(\tau_{1, s}, \tau_{2, s}\right) / \hbar} \\
& \times \operatorname{Im} \int^{\prime} d \vartheta_{1} d \vartheta_{2} \exp { }^{-\left.\frac{1}{2 \hbar} \sum_{i, j=1,2} \frac{\partial^{2} S\left(\tau_{1}, \tau_{2}\right)}{\partial \tau_{i} \partial \tau_{j}}\right|_{\tau_{i}=\tau_{i, s}} \vartheta_{i} \vartheta_{j}},
\end{aligned}
$$

where $\vartheta_{i}$ denotes deviations from the saddle point. Here we used $Z_{0} \approx 1$. The prime at the integral excludes the integration over the center of mass of the instanton. Finally we ignored here a Jacobian factor which describes the transition from the original field $\phi_{i}(\tau)$ to the instanton dimension $\tau$.

Below we will consider only two cases: either instantons of equal size appear at both impurities, corresponding to $\tilde{\phi}_{-}=0$, i.e. $\tau_{1, s}=\tau_{2, s} \equiv \tau_{s}$. This case will be called co-tunneling. Or there is only one instanton either on the left or the right impurity, corresponding to $\tilde{\phi}_{+}= \pm \tilde{\phi}_{-}$, i.e. $\tau_{1(2), s} \equiv \tau_{s}>0$ and $\tau_{2(1), s}=0$. This case will be called sequential tunneling. Using the results obtained previously for the single impurity ${ }^{8}$ case, the instanton action at $T=0$ can be written down immediately. It takes the form

$$
\begin{aligned}
\frac{S_{\text {inst }}}{\hbar}= & \frac{2 S_{\mathrm{kink}}}{\hbar}\left(\Theta_{H, \delta}\left(\tau_{1}\right)+\Theta_{H, \delta}\left(\tau_{2}\right)\right)+\frac{2}{K}\left[f\left(\tau_{1}\right)+f\left(\tau_{2}\right)\right] \\
& -\frac{e V_{0}}{2 \hbar}\left(\tau_{1}+\tau_{2}\right)+\left|\tau_{1}-\tau_{2}\right| E_{\operatorname{sign}\left(\tau_{1}-\tau_{2}\right)} \frac{1}{\hbar},
\end{aligned}
$$

where $\Theta_{H, \delta}(x)$ is a step function of width $\delta$. The different terms have the following meaning: $S_{\text {kink }}$ denotes the action of a kink, while $t=e^{-S_{\mathrm{kink}} / \hbar}$ is the tunneling transparency of a single impurity. The next term in (21) includes the kink-antikink interaction with

$$
f(\tau)=\int_{0}^{\omega_{c}} d \omega \frac{\pi}{I_{+}(\omega) \omega^{2}}[1-\cos (\omega \tau)] .
$$

The following voltage term describes the decrease of the energy by transferring an electron from the left to the quantum dot and from there to the right. Note that the voltage applied to at the ends of the system is, in general, different from the voltage at the impurities. However, if the wire is not too long and the impurities are strong, both voltages are approximately the same. Finally, the last term is the contribution from the Coulomb blockade. Going over to dimensionless time variable $\tau K e V_{0} / \hbar=y$ the action can be rewritten in the form

$$
\begin{aligned}
& \frac{S\left(y_{1}, y_{2}\right)}{\hbar}=\frac{2 S_{\mathrm{kink}}}{\hbar}\left[\Theta_{H, \delta}\left(y_{1}\right)+\Theta_{H, \delta}\left(y_{2}\right)\right] \\
& +\frac{2}{K}\left[F\left(y_{1}\right)+F\left(y_{2}\right)\right]-\frac{1}{2 K}\left(y_{1}+y_{2}\right)+\frac{1}{K}\left|y_{1}-y_{2}\right| \Delta X
\end{aligned}
$$

where

$$
F(y)=\int_{0}^{y Z} \frac{d \Omega}{\Omega} \frac{\sqrt{1+\frac{y Y}{|\Omega|}}(1-\cos \Omega)}{1+e^{-\frac{\pi \Omega}{y X}} \sqrt{1+\frac{y Y}{|\Omega|}}}
$$

with

$$
X=\frac{E_{c}}{e V_{0}}, \quad Y=\frac{\Gamma}{K e V_{0}}, \quad Z=\frac{\hbar \omega_{c}}{K e V_{0}} .
$$

Here we have used the ratios $X, Y, Z$ of the relevant energy scales of the problem. To calculated the integral we will assume that always $1, X, Y \ll Z$, i.e. $K e V_{0}, \Gamma, K E_{c} \ll \hbar \omega_{c}$. The integral can be approximated by the replacement $1-\cos \Omega \approx \Theta_{H}(\Omega-1)$. The calculation is done in Appendix $\mathrm{B}$ and gives the final result (B6). 


\section{Finite Temperatures}

So far we considered the case of zero temperature. At low but finite temperature the action and its saddle points are essentially unchanged, as long as the saddle point for $\tau$ is smaller than $\hbar / T$. For larger $T$ the tunneling rate is determined from the maximum of the action taken at $\tau=\hbar / T^{13}$. Again, for sequential tunneling one of the saddle points of $\tau_{i, s}$ vanishes. For the further discussion it is convenient, instead of (25) to introduce the following dimensionless parameters

$$
X_{T} \equiv \frac{K E_{c}}{T}, \quad Y_{T} \equiv \frac{\Gamma}{T}, \quad Z_{T} \equiv \frac{\hbar \omega_{c}}{T} .
$$

Accordingly, the dimensionless imaginary time is redefined as $z=\tau T / \hbar$. This gives, instead of (23), for the instanton action

$$
\begin{aligned}
& \frac{S\left(z_{1}, z_{2}\right)}{\hbar}=\frac{2 S_{\mathrm{kink}}}{\hbar}\left[\Theta_{H, \delta}\left(z_{1}\right)+\Theta_{H, \delta}\left(z_{2}\right)\right]-\frac{e V_{0}}{2 T}\left(z_{1}+z_{2}\right) \\
&+\frac{2}{K}\left[F_{T}\left(z_{1}\right)+F_{T}\left(z_{2}\right)\right]+\left|z_{1}-z_{2}\right| \Delta \frac{X_{T}}{K}
\end{aligned}
$$

where $F_{T}(z)$ is given by (D1). Note that here either $z_{1}=z_{2}=1$ for co-tunneling or $z_{1}=1$ and $z_{2}=0$ (or vice versa) for sequential tunneling.

\section{E. Cross-Over between Sequential and Co-Tunneling}

As mentioned already, to find the tunneling rate and hence the current $I$, we have to calculate the saddle points $y_{1, s}, y_{2, s}$ of (23). At $T=0$ the result depends on $X, Y, Z$ as well as on $\Delta$ and $K$. All terms in (23) are symmetric in $y_{1}, y_{2}$ apart from the last one which determines the difference between $y_{1}$ and $y_{2}$. These saddle points are calculated in Appendix C in Eqs. (C1) and (C2), respectively. To find the cross-over line between sequential tunneling and co-tunneling we have to equate the saddle point action of the two cases:

$$
2 F\left(y_{s}\right)-\left(\frac{1}{2}-\Delta X\right) y_{s}=\frac{2 K S_{\mathrm{kink}}}{\hbar}+4 F\left(y_{c}\right)-y_{c}
$$

As it is shown in Appendix C the cross-over between sequential tunneling and co-tunneling happens at

$$
X_{c} \equiv \frac{E_{c}}{e V_{0}}=\frac{1}{2 \Delta} \begin{cases}\frac{1}{1+2 Y_{1}}, & \frac{\Gamma}{K e V_{0}}=Y \leq 1 \\ 1-\frac{1}{2+K S_{\mathrm{kink}} /(\hbar \pi Y)}, & \frac{\Gamma}{K e V_{0}}=Y \gg 1\end{cases}
$$

where $Y_{1} \equiv\left(\frac{t^{2 K}}{2 \Delta Z^{2}}\right)^{\frac{1}{1-K}}$. The cross-over line is depicted in Fig. 1.

Next we calculate the crossover between co-tunneling and sequential tunneling for finite temperatures. In this case the crossover condition corresponding to (28) is given by

$$
\frac{2}{K} F_{T}(1)+\Delta \frac{X_{T}}{K}=\frac{2 S_{\mathrm{kink}}}{\hbar}+\frac{4}{K} F_{T}(1) .
$$

To solve this equation, we start with the regime $\Gamma, E_{c} K \ll T$, in which $F_{T}(1)$ is given by the expression case $\mathrm{i}$ in formula (D1). In this regime Eq. (30) leads to

$$
\Delta X_{T}=\frac{2 K S_{\mathrm{kink}}}{\hbar}+2 \ln Y_{T} \gg 2
$$

This violates the starting condition $\Gamma, E_{c} K \ll T$. Thus the crossover between the sequential tunneling and cotunneling is not possible in this regime. Similarly, it can be shown that the crossover cannot happen in the regime $X_{T}^{2} / Y_{T} \ll 1 \ll Y_{T}$. In the remain three regimes we find self-consistent solutions for the crossover. These results are summarized by the following expression

$$
\Delta X_{T} \approx \begin{cases}2 K S_{\mathrm{kink}} / \hbar, & Y_{T} \ll 1 \ll X_{T}, \\ 2 K S_{\mathrm{kink}} / \hbar+\sqrt{2 \pi}\left(\sqrt{Y_{T}}-1\right), & 1 \ll Y_{T} \ll X_{T}, \\ 2 K S_{\mathrm{kink}} / \hbar+\left[\sqrt{2 \pi Y_{T}}+\sqrt{2 \pi}\left(Y_{T} / X_{T}-2\right)\right], & 1 \ll X_{T}^{2} / Y_{T} \ll Y_{T}\end{cases}
$$

The various regimes and crossovers between them for finite temperature are illustrated in Fig. 2 ,

\section{CO-TUNNELING}

\section{A. Zero temperature}

In this section we will consider co-tunneling. In this case the instanton covers both impurities, $y_{1}=y_{2}=y_{c}$, and the electron will tunnel in one step through them.

In the regime of very weak dissipation, $\Gamma \ll K e V_{0}$, i.e. 


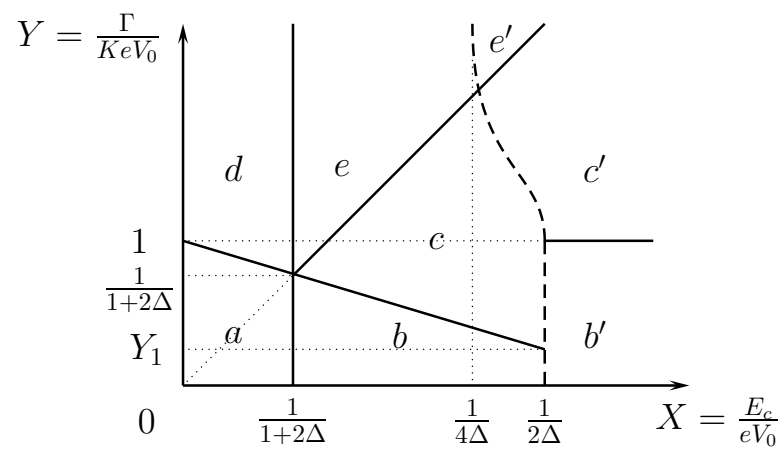

FIG. 1: Cross-over diagram at zero temperature: The regions $(a)$ - (e) correspond to sequential tunneling, the regions $\left(b^{\prime}\right)$, $\left(c^{\prime}\right)$ and $\left(e^{\prime}\right)$ to co-tunneling. The dashed cross-over line is given by Eq. (29)

regime $\left(b^{\prime}\right)$, we get

$$
I \sim t^{4} \omega_{\delta}\left(\frac{K E_{c}}{\hbar \omega_{c}}\right)^{\frac{2}{K}}\left(\frac{e V_{0}}{\hbar \omega_{c}}\right)^{\frac{2}{K}-1}, \quad \Gamma \ll K e V_{0} \ll K E_{c}
$$

where $\omega_{\delta} \equiv 1 /\left(\omega_{c} \delta^{2}\right)$ and $\delta \sim 1 / U$ is a short time cut-off. This result is similar to the dissipation free case considered for a single impurity by Kane and Fisher ${ }^{3.4}$. This is intuitively expected since in the co-tunneling process the island can be effectively viewed as a "big" impurity with renormalized strength. The factor $t^{4}$ corresponding to the tunneling through two impurities. Such a $t^{4}$ prefactor has been found previously in a study of Coulomb blockade in a system with long range interaction 14 . For $K>1$ the conductance $G=I / V_{0}$ diverges according to (33) which signals the approach to the perfect conductance $G=e^{2} / h$.

In the opposite regimes $\left(c^{\prime}\right)$ and $\left(e^{\prime}\right)$ of strong dissipation, we find

$$
\begin{gathered}
I \sim t^{4} \omega_{\delta} e^{-\frac{2 \pi \Gamma}{K^{2} e V_{0}}}\left(\frac{\Gamma}{e V_{0}}\right)^{\frac{3}{2}}\left(\frac{\Gamma}{\hbar \omega_{c}}\right)^{\frac{4}{K}-1}\left(e^{\frac{\sqrt{2 \pi} \Gamma}{E_{c} K}}-1\right)^{-\frac{2}{K}}, \\
K e V_{0} \ll \Gamma, K E_{c} .
\end{gathered}
$$

Again, this result has the same voltage-dependence as those of the single-impurity cases ${ }^{8}$. As it follows from (34), dissipation strongly reduces the tunneling probability through the impurities. The last factor is an interpolation formula between the cases $\Gamma \ll K E_{c}$ and $\Gamma \gg K E_{c}$, respectively. Since this factor appears as well in the formulas below, $t^{2}\left(e^{\frac{\sqrt{2 \pi} \Gamma}{E_{c} K}}-1\right)^{-\frac{1}{K}} \approx t^{2} e^{-\sqrt{\frac{2}{\pi}} \frac{a}{K L_{\eta}}}$ can be considered as the effective transmission coefficient of the impurity in the case of strong dissipation.

\section{B. Finite temperatures}

For nonzero temperature, besides $K E_{c}, \Gamma, \hbar \omega_{c}$ and $K e V_{0}$, the temperature $T$ appears as a new energy scale.

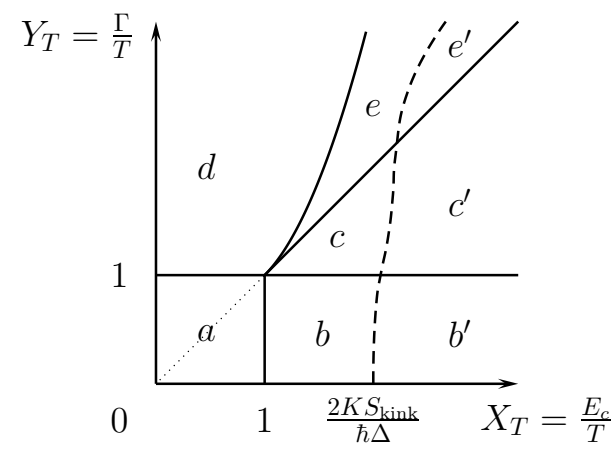

FIG. 2: Cross-over diagram at nonzero temperature: The regions $(a)-(e)$ correspond to sequential tunneling, the regions $\left(b^{\prime}\right),\left(c^{\prime}\right)$ and $\left(e^{\prime}\right)$ to co-tunneling. The dashed cross-over line is given by Eq. (32).

This allows in general a large number of different regimes. In the following we will therefore restrict ourselves to the case where $K e V_{0}$ is smaller than all other energies.

In the limit of weak dissipation (regime $\left(b^{\prime}\right)$ ) we get for the conductance

$$
G \sim t^{4} \omega_{\delta}\left(\frac{T}{\hbar \omega_{c}}\right)^{\frac{2}{K}-2}, \Gamma \ll T \ll K E_{c}
$$

which again agrees, apart from the factor $t^{4}$, with the result of Kane and Fisher ${ }^{\underline{3}}$ in the dissipation free case. In the opposite regime $\left(e^{\prime}\right)$ of strong dissipation we get instead

$$
G \sim t^{4} \omega_{\delta} e^{-\sqrt{\frac{8 \pi \Gamma}{K^{2} T}}}\left(e^{\frac{\sqrt{2 \pi} \Gamma}{E_{c} K}}-1\right)^{-\frac{2}{K}}, T \ll K E_{c}, \Gamma .
$$

It should be noted that the present approach does not allow the precise determination of the numerical prefactors in the exponential terms. The leading temperature dependence is the same as for the single impurity case, see Ref.

\section{SEQUENTIAL TUNNELING}

\section{A. Zero temperatures}

Let us next consider the resonant case $\Delta=0$ where the cross-over line between sequential and co-tunneling moves to $E_{c} \rightarrow \infty$. Then, according to (13), starting with the ground state $Q=m$, it does not cost energy to add a particle to the quantum dot. To remove it from the quantum dot in the new state $Q=m+1$ does not cost energy as well. The tunneling rate for each process is the same and follows from the saddle point of (23) with $y_{2}=0$ and $y_{1}=y_{s}$. Below we will present more general results for the case which includes a weak deviation from perfect resonance.

If $0<\Delta \ll 1$, bringing an electron to the quantum dot costs an energy $\Delta E_{c}$ whereas in the second step, in 
which the electron leaves the dot, this energy is again released. Thus tunneling through the dot is dominated by the first step. Similarly, if $\Delta<0$, it costs first an energy $|\Delta| E_{c}$ to bring an electron out of the quantum dot whereas in the second step a second electron tunnels from the left into the dot, which is accompanied by an energy gain $-|\Delta| E_{c}$. Thus, again the second process is faster than the first one, the latter dominates the tunneling probability. Both cases can be combined by replacing $\Delta$ by its absolute value.

Plugging the expressions for the saddle points (C1) into the action we get for the current in the regime $(a)$ of large voltage

$$
I=G V_{0} \sim t^{2} \omega_{\delta}\left(\frac{e V_{0}}{\hbar \omega_{c}}\right)^{\frac{2}{K}-1}, \quad \Gamma, K E_{c} \ll K e V_{0} .
$$

This corresponds to non-dissipative incoherent sequential tunneling and has the same voltage dependence as the single impurity tunneling in the absence of dissipation ${ }^{3}$. The critical $K$-value for the conductance $G$ is $K=1$. For $K>1$ the conductance increases for decreasing voltage signaling a perfect conductance in the zero voltage limit. It should however be taken into account that this limit cannot be performed because of the restriction $\mathrm{KeV}_{0} \gg$ $\Gamma$.

In the opposite limit of very low voltage (regimes $(c)$ and $(e)$ ) we get instead

$$
\begin{aligned}
I \sim t^{2} \omega_{\delta} e^{-\frac{\pi \Gamma}{K^{2}\left(e V_{0}-2|\Delta| E_{c}\right)}}\left(\frac{\hbar \omega_{c}}{e V_{0}-2|\Delta| E_{c}}\right)^{\frac{3}{2}} \\
\quad \times\left(e^{\frac{\sqrt{2 \pi} \Gamma}{E_{c} K}}-1\right)^{-\frac{1}{K}}, K\left(e V_{0}-2|\Delta| E_{c}\right) \ll K E_{c}, \Gamma .
\end{aligned}
$$

In these cases for $|\Delta|=0$ the system shows dissipative resonant tunneling. In comparison with the corresponding result for the co-tunneling (34), the expressions in (38) are larger by an exponent $1 / 2$ in the leading voltage dependence (provided $\Delta \rightarrow 0$ ). Clearly, for all values of $K$ dissipation is dominant and reduces the current strongly.

Finally, there are the intermediate cases $(b)$

$$
\begin{array}{r}
I \sim t^{2} \omega_{\delta}\left(\frac{K E_{c}}{\hbar \omega_{c}}\right)^{\frac{1}{K}}\left(\frac{e V_{0}-2|\Delta| E_{c}}{\hbar \omega_{c}}\right)^{\frac{1}{K}-1}, \\
\frac{\Gamma}{K} \ll e V_{0}-2|\Delta| E_{c} \ll E_{c}
\end{array}
$$

and $(d)$

$I \sim t^{2} \omega_{\delta}\left(\frac{\Gamma}{\hbar \omega_{c}}\right)^{\frac{2}{K}-1}\left(\frac{\Gamma}{e V_{0}}\right)^{\frac{3}{2}} e^{-\frac{4 \pi \Gamma}{K^{2} e V_{0}}}, E_{c} \ll e V_{0} \ll \frac{\Gamma}{K}$.

In case (b), Eq. (39), under resonant conditions, $|\Delta|=0$, the conductance $G=I / V_{0}$ diverges for $V_{0} \rightarrow 0$ and $1 / 2<K$, signaling a perfect conductance. For small but finite $\Gamma$ however, the conductance is limited by
$G \sim t^{2} \omega_{\delta}\left(\Gamma / \hbar \omega_{c}\right)^{\frac{1}{K}-2}$. Case $(d)$ corresponds to dissipative incoherent sequential tunneling. In comparison with the dissipative single impurity result ${ }^{8}$, the expressions in (40) are smaller by an exponent 2 in the leading voltage dependence.

Since in regimes $a$ and $d$ the tunneling through the two impurities is independent, the total conductance can be calculated by the formula for two identical conductances connected in series, $G=G_{s}\left(V_{0} / 2\right) / 2$, where $G_{s}\left(V_{0} / 2\right)$ denotes the conductance for single impurity with voltage drop $V_{0} / 2$. This also leads to results (37) and (40).

One can notice that in some formulas (e.g. (34), (40)) no factor $\Delta$ appears, while in (38) and (39) it appears. The reason is that the latter two equations correspond to regimes $(b),(c)$ and $(e)$, see Fig. 1. In these regimes, $2 \Delta E_{c}$ can be arbitrary close to $e V_{0}$ when one is close to the crossover between sequential tunneling and cotunneling. Formula (40) corresponds to regime $(d)$, which is always far away from the crossover. This implies that $\Delta E_{c}$ is always much smaller than $e V_{0}$, and hence neglected in (40). Eq. (34) is for co-tunneling regime where the Coulomb blockade term (i.e, the term that involves $\Delta$ in Eq. (23) ) disappears.

\section{B. Finite Temperature}

At finite temperature we obtain various tunneling regimes which have one to one correspondence to those at zero temperature. Moreover, the discussions for these regimes apply in both cases of zero and nonzero temperatures. Therefore, to avoid unnecessary repetition we only give results for finite temperature. We obtain for the conductance in regime $(a)$

$$
G \sim t^{2}\left(\frac{T}{\hbar \omega_{c}}\right)^{\frac{2}{K}-2}, \quad \Gamma, E_{c} K \ll T,
$$

which has the same temperature dependence as the single impurity in the absence of dissipation ${ }^{3}$.

At low temperature we obtain the result of dissipative resonant tunneling of regimes $(c)$ and $(e)$

$$
\begin{array}{r}
G \sim t^{2} e^{-\frac{E_{c}|\Delta|}{T}} A_{3}(T) e^{-\sqrt{\frac{2 \pi \Gamma}{K^{2} T}}}\left(e^{\frac{\sqrt{2 \pi} \Gamma}{E_{c} K}}-1\right)^{-\frac{1}{K}}, \\
T \ll K E_{c}, \frac{\left(K E_{c}\right)^{2}}{\Gamma}, \Gamma .
\end{array}
$$

$A_{3}(T)$ is some power-law temperature-dependent function, which is subdominant to the exponential temperature-dependent part in (42).

At intermediate temperature we find in regime $(b)$

$$
G \sim t^{2}\left(\frac{K E_{c}}{\hbar \omega_{c}}\right)^{\frac{1}{K}} e^{-\frac{E_{c}|\Delta|}{T}}\left(\frac{T}{\hbar \omega_{c}}\right)^{\frac{1}{K}-2}, \Gamma \ll T \ll K E_{c} .
$$

Thus the conductance in regimes $(c),(e)$ and $(b)$ is exponentially suppressed away from resonance. At resonance 
(i.e, $|\Delta|=0$ ) the conductance in regime $(b)$ increases with decreasing temperature if $K>1 / 2$, signaling perfect conductance if $\Gamma=0$. For finite dissipation the conductance reaches a saturation value $\sim t^{2}\left(\Gamma / \hbar \omega_{c}\right)^{\frac{1}{K}-2}$. Finally in region $(d)$ we get

$$
G \sim t^{2} A_{4}(T) e^{-\sqrt{\frac{8 \pi \Gamma}{K^{2} T}}}, \quad \frac{\left(K E_{c}\right)^{2}}{\Gamma} \ll T \ll \Gamma
$$

where $A_{4}(T)$ is again some power-law temperaturedependent function.

\section{CONCLUSIONS}

In the present paper we have calculated the conductance $G$ of a dissipative Luttinger liquid with a quantum dot formed by two strong impurities, using an instanton approach. The following results have been obtained:

(i) Depending on the ratio of the Coulomb energy of the quantum dot, $|\Delta| E_{c}$, and the temperature $T$ (or the voltage drop $e V_{0}$, respectively), there is a crossover from co-tunneling for low temperatures (or small applied voltage) to sequential tunneling for larger temperatures (or voltage). At resonance, $|\Delta|=0$, the region for cotunneling disappears completely and the conductance is always due to sequential tunneling. The cross-over lines between co-tunneling and sequential tunneling are given by Eqs. (29) and (32), respectively (compare also Fig. 1 and Fig. (2).

(ii) If the voltage drop through the impurities $e V_{0}$ is much smaller than all other energy scales, the response of the system is linear. Then for very weak dissipation, $\Gamma \ll K E_{c}$, and $1 / 2<K<1$, the conductance at resonance, $|\Delta|=0$, shows a minimum at $T \approx K E_{c}$ between the regimes (a) and (b) decribed by formulas (41) and (43). This result agrees with the findings of Furusaki and Nagaosa ${ }^{6}$. For very low temperatures, $T<\Gamma$, however, the conductance drops exponentially due to the dissipation (see Eq. (42) and Fig. 3.)

Off resonance, for $|\Delta| E_{c}>T$, the conductance is exponentially suppressed even at larger temperatures, see (43). This reduction of $G$ is limited however by the crossover to co-tunneling, see Eq. (33).

In the opposite limit of strong dissipation, $\Gamma \gg K E_{c}$, the conductance drops to exponentially small values as soon as $T \ll \Gamma$ (see Eqs. (42) and (44)).

(iii) At $T=0$ and at resonance, $\Delta=0$, the voltage dependent conductance $G=I / V_{0}$ shows a behavior similar to that of the temperature dependent conductance, as follows from eqs. (37) - (40). There is again a non-monotonic behavior for $\Gamma<K E_{c}$ and a monotonic behavior for $\Gamma>K E_{c}$ (see also Fig. [3).

\section{Acknowledgements}

This work is financially supported by Sonderforschungsbereich 608. We thank R. Citro and M. Fogler

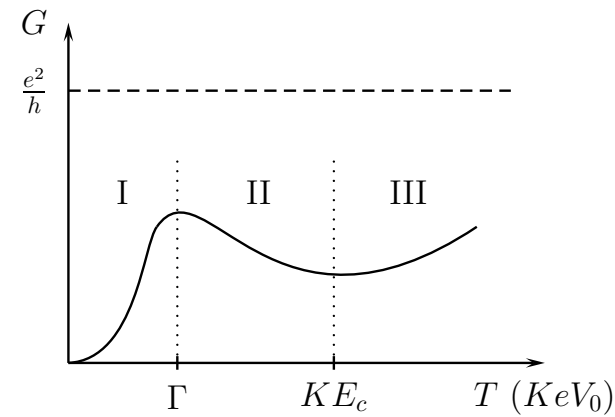

FIG. 3: Conductance as a function of temperature (voltage) for the case of weak dissipation $\Gamma \ll K E_{c}$.

for discussions in an early stage of this project.

\section{APPENDIX A: LIST OF SYMBOLS}

\begin{tabular}{lll}
\hline \hline Symbol & Definition & Quantity \\
\hline$K$ & & Luttinger liquid parameter \\
$v$ & & $\begin{array}{l}\text { excitation velocity } \\
\text { dissipation strength }\end{array}$ \\
$\eta$ & & high frequency cutoff \\
$\omega_{c}$ & & spacing between impurities \\
$a$ & & external voltage \\
$V_{0}$ & & temperature \\
$T$ & & tunneling transparency \\
$t$ & & compressibility \\
$\kappa$ & $K /(\pi \hbar v)$ & charging energy of the dot \\
$E_{c}$ & $\pi \hbar v /(K a)$ & distance to the resonance \\
$\Delta$ & $n+\frac{1}{2}-\frac{k_{F} a}{\pi}$ & damping of plasmons \\
$\Gamma$ & $\hbar v K \eta$ & \\
$X$ & $E_{c} /\left(e v_{0}\right)$ & \\
$Y$ & $\Gamma /\left(K e V_{0}\right)$ & \\
$Z$ & $\hbar \omega_{c} /\left(K e V_{0}\right)$ & \\
$X_{T}$ & $K E_{c} / T$ & \\
$Y_{T}$ & $\Gamma / T$ & \\
$Z_{T}$ & $\hbar \omega_{c} / T$ & \\
\hline \hline
\end{tabular}

\section{APPENDIX B:}

In this appendix we briefly describe how to calculate the instanton action for the interaction between the kink and antikink [i.e., $F(y)$ given by formula (24)]. The integrant of the integral on the right hand side of formula (24) shows several crossovers in $\Omega$-space. These crossovers can be obtained by equating $Y y / \Omega$ and the exponent of the exponential to 1 , separately. For $Y \ll X$, the crossovers are at $\Omega=Y y$ and $\Omega=X y$, which separates the three regions $\Omega \ll Y y \ll X y, Y y \ll \Omega \ll X y$, and $Y y \ll X y \ll \Omega$. For $Y \gg X$, the crossovers are at $\Omega=Y y$ and $\Omega=X^{2} y / Y$, which again separates three 
regions $\Omega \ll X^{2} y / Y \ll Y y, X^{2} y / Y \ll \Omega \ll Y y$ and $X^{2} y / Y \ll Y y \ll \Omega$. To evaluate the integral we dissemble it into small ones according to the different regions separated by the crossovers. We consider the two cases $Y \ll X$ and $Y \gg X$, separately. We start with $Y \ll X$. For $1 \gg X y \gg Y y$, we have

$$
F(y) \approx \int_{0}^{Z y} d \Omega \frac{1-\cos \Omega}{\Omega}=\ln (Z y) .
$$

For $Y y \ll 1 \ll X y$, we have

$$
\begin{aligned}
F(y) & \approx \frac{1}{2} \int_{0}^{X y} d \Omega \frac{1-\cos \Omega}{\Omega}+\int_{X y}^{Z y} d \Omega \frac{1-\cos \Omega}{\Omega} \\
& =\frac{1}{2} \ln (X y)+\ln \left(\frac{Z}{X}\right) .
\end{aligned}
$$

For $1 \ll y Y \ll y X$, we have

$$
\begin{aligned}
F(y) & \approx \frac{1}{2} \int_{0}^{Y y} d \Omega \frac{\sqrt{Y y \Omega}}{\Omega^{2}}(1-\cos \Omega) \\
& +\frac{1}{2} \int_{Y y}^{X y} d \Omega \frac{1-\cos \Omega}{\Omega}+\int_{X y}^{Z y} d \Omega \frac{1-\cos \Omega}{\Omega} \\
& =\frac{1}{2}\left[\sqrt{2 \pi}(\sqrt{Y y}-1)+\ln \left(\frac{X}{Y}\right)+2 \ln \left(\frac{Z}{X}\right)\right] .
\end{aligned}
$$

$$
F(y)= \begin{cases}\ln (Z y), & \text { (i) } y Y, y X \ll 1, \\ \frac{1}{2} \ln \left(Z^{2} y / X\right), & \text { (ii) } y Y \ll 1 \ll y X, \\ \frac{1}{2}\left[\sqrt{2 \pi Y y}+\ln \left(\frac{Z^{2}}{X Y}\right)\right], & \text { (iii) } 1 \ll y Y \ll y X, \\ \sqrt{2 \pi Y y}+\ln \left(\frac{Z}{Y}\right), & \text { (iv) } y X^{2} / Y \ll 1 \ll y Y, \\ \frac{1}{2}\left[\sqrt{2 \pi Y y}+\sqrt{2 \pi} \frac{Y}{X}+2 \ln \left(\frac{Z}{Y}\right)\right], & \text { (v) } 1 \ll y X^{2} / Y \ll y Y .\end{cases}
$$

\section{APPENDIX C:}

The saddle points for sequential and co-tunneling are given by (C1) and (C2), respectively.

$$
y_{s}=\left\{\begin{array}{lll}
\frac{4}{1-2 \Delta X}, & \text { (a) } Y+2 \Delta X, X(1+2 \Delta) \ll 1, \\
\frac{2}{1-2 \Delta X}, & \text { (b) } Y+2 \Delta X \ll 1 \ll X(1+2 \Delta), \\
\frac{2 \pi Y}{(1-2 \Delta X)^{2}}, & \text { (c) } 1 \ll Y+2 \Delta X \ll X(1+2 \Delta), \\
\frac{8 \pi Y}{(1-2 \Delta X)^{2}}, & \text { (d) } X(1+2 \Delta) \ll 1 \ll Y+2 \Delta X, \\
\frac{2 \pi Y}{(1-2 \Delta X)^{2}}, & \text { (e) } 1 \ll X(1+2 \Delta) \ll Y+2 \Delta X .
\end{array}\right.
$$

Here the different areas of validity $(a)-(e)$ are separated by the lines $X=Y, Y=1-2 \Delta X$ and $X=1 /(1+$ $2 \Delta$ ) (see also Fig. 1). These saddle points have to be
Now we discuss the case $Y \gg X$. For $1 \gg Y y \gg$ $X^{2} y / Y$, one also gets the result formula (B1). For $X^{2} y / Y \ll 1 \ll Y y$, one gets

$$
\begin{aligned}
F(y) & \approx \int_{0}^{Y y} d \Omega \frac{\sqrt{Y y \Omega}}{\Omega^{2}}(1-\cos \Omega)+\int_{Y y}^{Z y} d \Omega \frac{1-\cos \Omega}{\Omega} \\
& =\sqrt{2 \pi}(\sqrt{Y y}-1)+\ln \left(\frac{Z}{Y}\right) .
\end{aligned}
$$

For $1 \ll X^{2} y / Y \ll Y y$, one finds

$$
\begin{aligned}
F(y) & \approx \frac{1}{2} \int_{0}^{X^{2} y / Y} d \Omega \frac{\sqrt{Y y \Omega}}{\Omega^{2}}(1-\cos \Omega) \\
+ & \int_{X^{2} y / Y}^{Y y} d \Omega \frac{\sqrt{Y y \Omega}}{\Omega^{2}}(1-\cos \Omega)+\int_{Y y}^{Z y} d \Omega \frac{1-\cos \Omega}{\Omega} \\
& =\frac{1}{2}\left[\sqrt{2 \pi Y y}+\sqrt{2 \pi}\left(\frac{Y}{X}-2\right)+2 \ln \left(\frac{Z}{Y}\right)\right] .
\end{aligned}
$$

Finally, the results can be summarized by . 
The crossover between the sequential tunneling and the co-tunneling is defined as the point at which the currents for the two different tunneling mechanism are equal. Assuming $\Delta \ll 1$, in Sec. IIE it is pointed out that the crossover between the co-tunneling and the sequential tunneling in regimes $a$ and $d$ [i.e., $X \gg 1 /(1+2 \Delta)$ ] cannot happen. This is clearly illustrated in Fig. 1.

We start with very small $Y$ (i.e., $Y<Y_{1}$ ) such that near the crossover the currents for the sequential tunneling and the co-tunneling are given by formulae (39) for regime $b$ and (35) for regime $b^{\prime}$, respectively. The value of $Y_{1}$ will be determine afterwards. After a straightforward calculation the crossover is found to be

$$
X=\frac{1-\left(\frac{t^{2 K}}{2 \Delta Z^{2}}\right)^{\frac{1}{1-K}}}{2 \Delta} \approx \frac{1}{2 \Delta} .
$$

However, this result is self-consistent only if the current for the sequential tunneling is indeed given by formula (39) for regime $b$ [i.e., $Y+2 \Delta X \ll 1 \ll X(1+2 \Delta)$ ]. This leads to a restriction on the validity of (C3)

$$
Y \ll Y_{1} \equiv\left(\frac{t^{2 K}}{2 \Delta Z^{2}}\right)^{\frac{1}{1-K}} .
$$

$Y_{1}$ is essentially very small.

For $Y$ slightly bigger than $Y_{1}$, the current for the sequential tunneling is given by formula (38) for regime $c$, while the current for the co-tunneling remains in regime $b^{\prime}$. In this situation the crossover is determined by

$$
\begin{aligned}
& t^{2} \omega_{\delta}\left(\frac{Y}{Z}\right)^{\frac{2}{K}+\frac{1}{2}}\left(\frac{X}{Y}\right)^{\frac{1}{K}} Z^{\frac{3}{2}}(1-2 \Delta X)^{-\frac{3}{2}} \\
& \times \exp \left(-\frac{\pi Y}{K(1-2 \Delta X)}\right)=t^{4} \omega_{\delta}\left(\frac{X}{Z}\right)^{\frac{2}{K}}\left(\frac{1}{Z}\right)^{\frac{2}{K}-1}
\end{aligned}
$$

where we have chosen to express the currents in terms of the ratios of relevant energy scales $X, Y$ and $Z$. After some algebra we obtain

$$
X \approx \frac{1-\frac{\hbar \pi Y}{2 K S_{\mathrm{kink}}}}{2 \Delta} \approx \frac{1}{2 \Delta} .
$$

This result is valid for $Y_{1} \ll Y \ll 1$.

Now for $1 \ll Y \ll X$, the current for the sequential tunneling is still in regime $c$, while the one for the co-tunneling just moves into regime $c^{\prime}$ and is given by formula (34). In this regime the crossover is given by

$$
\begin{aligned}
& t^{2} \omega_{\delta}\left(\frac{Y}{Z}\right)^{\frac{2}{K}+\frac{1}{2}}\left(\frac{X}{Y}\right)^{\frac{1}{K}} Z^{\frac{3}{2}}(1-2 \Delta X)^{-\frac{3}{2}} \\
& \times \exp \left(-\frac{\pi Y}{K(1-2 \Delta X)}\right) \\
&=t^{4} \omega_{\delta}\left(\frac{Y}{Z}\right)^{\frac{4}{K}+\frac{1}{2}}\left(\frac{X}{Y}\right)^{\frac{2}{K}} Z^{\frac{3}{2}} \exp \left(-\frac{2 \pi Y}{K}\right),
\end{aligned}
$$

which leads to

$$
\begin{aligned}
1-2 \Delta X & =\frac{\pi Y}{\frac{2 K S_{\text {kink }}}{\hbar}+2 \pi Y+\ln \left(\frac{Z^{3}}{X Y^{2}}\right)} \\
& \approx \frac{\pi Y}{\frac{2 K S_{\text {kink }}}{\hbar}+2 \pi Y} .
\end{aligned}
$$

This crossover implies that for $1 \ll Y \ll X$ the range of $X$ on the crossover is within $1 /(4 \Delta) \ll X \ll 1 /(2 \Delta)$. Thus, for $K S_{\text {kink }} / \hbar \ll 1 / \Delta$ the crossover intersects with the line $Y=X$ at $Y=X \approx 1 /(4 \Delta)$; otherwise they meet at $Y=X \approx 1 /(2 \Delta)$.

For $Y \gg X$, the current for the sequential tunneling and the co-tunneling are given by formulae (38) for regime $e$ and (34) for regime $e^{\prime}$. The crossover is given by

$$
\begin{aligned}
& t^{2} \omega_{\delta}\left(\frac{Y}{Z}\right)^{\frac{2}{K}+\frac{1}{2}} Z^{\frac{3}{2}}(1-2 \Delta X)^{-\frac{3}{2}} \\
& \times \exp \left(-\frac{\pi Y}{K(1-2 \Delta X)}\right) \exp \left(-\frac{\sqrt{2 \pi} Y}{K X}\right) \\
= & t^{4} \omega_{\delta}\left(\frac{Y}{Z}\right)^{\frac{4}{K}+\frac{1}{2}} Z^{\frac{3}{2}} \exp \left(-\frac{2 \pi Y}{K}\right) \exp \left(-\frac{2 \sqrt{2 \pi} Y}{K X}\right)
\end{aligned}
$$

This leads to

$$
\begin{aligned}
1-2 \Delta X & =\frac{\pi Y}{\frac{2 K S_{\text {kink }}}{\hbar}+2 \pi Y+\sqrt{2 \pi} \frac{Y}{X}+2 \ln \left(\frac{Z}{Y}\right)} \\
& \approx \frac{\pi Y}{\frac{2 K S_{\text {kink }}}{\hbar}+2 \pi Y} .
\end{aligned}
$$

Finally, the crossover between the sequential tunneling and the co-tunneling can be summarized as formula (29). The various regimes and the crossovers between them are illustrated in XY plane in Fig. 1.

\section{APPENDIX D:}

In this appendix we quote the final result for the function $F_{T}(z)$, which first appears in Eq. (27). It reads 


$$
F_{T}(z)= \begin{cases}\ln \left(Z_{T} z\right), & \text { (i) } z Y_{T}, z X_{T} \ll 1, \\ \frac{1}{2} \ln \left(Z_{T}^{2} z / X_{T}\right), & \text { (ii) } z Y_{T} \ll 1 \ll z X_{T}, \\ \frac{1}{2}\left[\sqrt{2 \pi Y_{T} z}+\ln \left(\frac{Z_{T}^{2}}{X_{T} Y_{T}}\right)\right], & \text { (iii) } 1 \ll z Y_{T} \ll z X_{T}, \\ \sqrt{2 \pi Y_{T} z}+\ln \left(\frac{Z_{T}}{Y_{T}}\right), & \text { (iv) } z X_{T}^{2} / Y_{T} \ll 1 \ll z Y_{T} \\ \frac{1}{2}\left[\sqrt{2 \pi Y_{T} z}+\sqrt{2 \pi} \frac{Y_{T}}{X_{T}}+2 \ln \left(\frac{Z_{T}}{Y_{T}}\right)\right], & \text { (v) } 1 \ll z X_{T}^{2} / Y_{T} \ll z Y_{T} .\end{cases}
$$

In the case of finite temperature, the previous saddle point solution for zero temperature and finite voltage does not apply when the distance between the kink and antikink is larger than the size of the imaginary time axis. Thus the maximum instanton action occurs at $\tau=\hbar / T$, i.e., $z=1$. Then the tunneling rate can be approximated as proportional to $\exp \left[-S\left(z_{1}=1, z_{2}=0\right) / \hbar\right]$ for the sequential tunneling and $\exp \left[-S\left(z_{1}=1, z_{2}=1\right) / \hbar\right]$ for the co-tunneling, respectively. Unlike in the case of zero temperature, the tunneling rates along both the voltage-favored and -unfavored directions are comparable. Therefore, the current should be proportional to the difference between these two. To the lowest order of $e V_{0}$, we get

$$
I \sim e V_{0} t^{2} \exp \left[-\frac{2}{K} F_{T}(1)-\frac{\Delta X_{T}}{K}\right]
$$

for the sequential tunneling and

$$
I \sim e V_{0} t^{4} \exp \left[-\frac{4}{K} F_{T}(1)\right]
$$

for the co-tunneling. This approximation is not accurate enough to give correct power-law temperaturedependence of the current. However, in the dissipative regime it captures the dominant exponential temperature-dependence. Plugging the expressions of $F_{T}(1)$ into the above two formulae, we obtain the results given in Secs. IVB and IIIB.
1 M.P.A. Fisher and L.I. Glazman, in Mesoscopic Electronic Transport, edited by L. Kouwenhoven (Kluwer, Dordrecht, 1997).

2 T. Giamarchi, Quantum Physics in One Dimension, (Clarendon Press, Oxford, 2003).

3 C.L. Kane and M.P.A. Fisher, Phys. Rev. Lett. 68, 1220 (1992).

4 C.L. Kane and M.P.A. Fisher, Phys. Rev. B 46, 7268 (1992).

5 C.L. Kane and M.P.A. Fisher, Phys. Rev. B 46, 15233 (1992).

6 A. Furusaki and N. Nagaosa, Phys. Rev. B 47, 3827 (1993).

7 M. A. Cazalilla, F. Sols and F. Guinea, Phys. Rev. Lett. 97, 076401 (2006).
8 Z. Ristivojevic and T. Nattermann, Phys. Rev. Lett. 101, 016405 (2008).

${ }^{9}$ F.D.M. Haldane, Phys. Rev. Lett. 47, 1840 (1981).

10 A. O. Caldeira and A. J. Leggett, Phys. Rev. Lett. 46, 211 (1981).

11 C. Callan and Coleman, Phys. Rev. D 16, 1762 (1977).

12 A. I. Larkin and Y. N. Ovchinnikov, Sov. Phys. JETP 59, 420 (1984).

13 T. Nattermann, T. Giamarchi and P. Le Doussal, Phys. Rev. Lett. 91, 056603 (2003).

14 H. Maurey and T. Giamarchi, Europhys. Lett. 38, 681 (1997). 